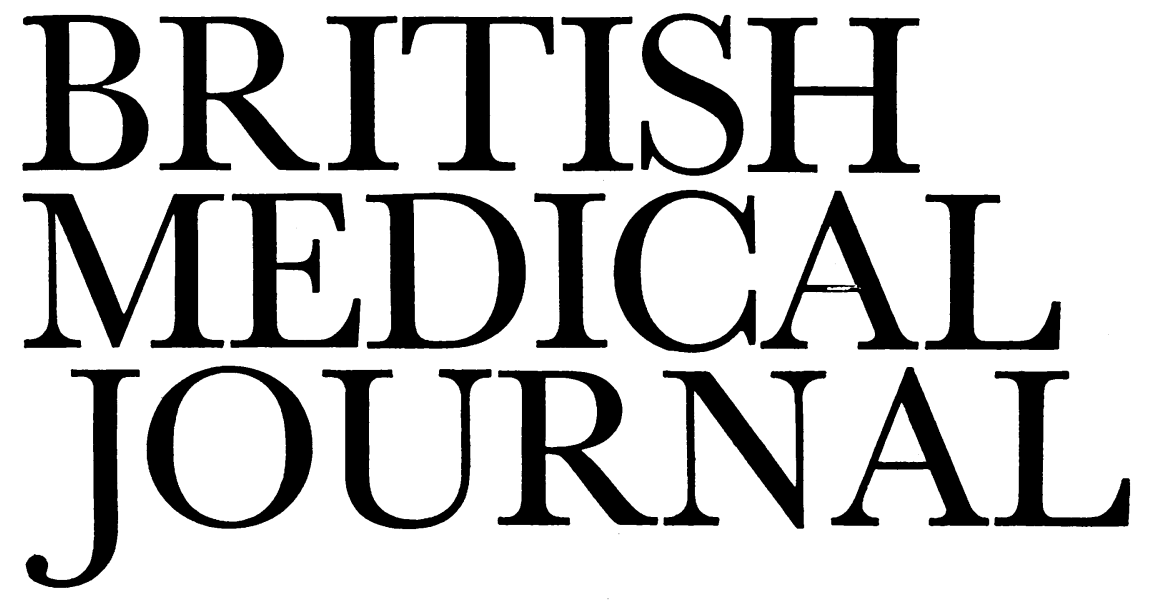

\title{
London's medicine
}

Doctors have grown weary of organisational reform: in the past decade they have had to struggle to continue their day-today work against the upheavals caused by the reorganisation of the NHS and the restructuring of nursing by Salmon, find their way through a maze of ever-proliferating committees and bureaucracy, and cope with a whole panoply of minor irritations such as the introduction of SI units. So a common initial response to Lord Flowers's proposals ( $p$ 731) for a new framework ${ }^{1}$ for medical education in London will be to ask why. Why more change?

On this occasion, however, the need for change is based on realities rather than on debatable theories. Firstly, the population of central London has fallen and is continuing to fall. The London Health Planning Consortium ${ }^{2}$ (see p 734) believes that in the next 10 years the number of acute beds in the teaching districts should be cut by $2300-$ a process already begun by the regional health authorities in response to the DHSS policy of reallocation of resources (RAWP). ${ }^{3}$ With fewer patients available, the undergraduate teaching hospitals have already begun either to move out of central London (the Charing Cross, St George's, and the Royal Free) or to make more use of outlying general hospitals for teaching.

Secondly, the University of London has found that medicine has taken an ever-increasing proportion of its budget-from $31 \%$ in $1969-70$ to $36 \%$ in $1978-9$. Medical schools have had their resources cut in real terms and staff vacancies have been frozen; but further economies seem unavoidable. Inevitably, therefore, the undergraduate teaching hospitals were going to have to find ways of accelerating their use of the periphery of London as their source of patients, while at the same time finding ways of co-operating to make more effective use of scarce resources. Lord Flowers's committee was asked to suggest the best way to reorganise medical education within that framework while maintaining existing targets of undergraduate education. Postgraduate institutes and education were also included in the committee's remit, which gave it the task-already attempted several times before-of rationalising the scattered units making up the 12 institutes of the British Postgraduate Medical Federation while causing as little damage as possible to their reputation for research and clinical excellence.

The committee made two decisions before getting down to detail. Firstly, it took the advice of the University of London Medical Group (representing medical students) that where a choice had to be made between close contact with their teaching hospital or education on the same site as other faculties students preferred the hospital contact-a decision that led to its verdict against King's College in the Strand and must have contributed to the verdict against the Westminster Medical School.

Secondly, the committee clearly believed that "large is beautiful." Its recommendations for amalgamations and fusions go further than many people expected, but Lord Flowers seems convinced that his proposals will be cost effective. Some amalgamation of medical schools has been accepted policy ever since Lord Todd's Royal Commission on Medical Education ${ }^{4}$ pointed to the absurdity of London University having 12 professorial chairs and 12 service departments in many of the main specialties. Of the six "pairings" proposed by Todd, ${ }^{4}$ only one has come into being, the link between the London Hospital and St Bartholomew's; but even so that experiment has shown that joint departments can function effectively. Rather than following the symmetry of the Todd pairings, however, the Flowers Committee chose to take a pragmatic approach. It looked at the geographical clustering of undergraduate and postgraduate hospitals and their associated schools and institutes, the quality of their buildings, the population served, and the transport links between them. Taking full account of the conclusions on service needs reached by the London Planning Consortium, it then proposed groupings that made practical sense in the current economic climate. In one case it grouped together University College Hospital, the Middlesex, and the Royal Free, four of the postgraduate institutes, and the London School of Hygiene and Tropical Medicine; at the other extreme it left the new Charing Cross Medical School alone, except for links with the Cardiovascular Institute and the Institute of Obstetrics and Gynaecology.

In contrast with earlier proposals for the reorganisation of London medicine, the Flowers framework seems likely to be carried through in some form-simply because the University of London holds the purse strings. The report itself confidently states that "those institutions for which we have recommended closure should not accept new students after 1981-2," and that places for 1982-3 should be offered on the basis of the new arrangements. Lord Flowers also acknowledges that uncertainty in the minds of staff lowers efficiency and morale, and one complete chapter of the report discusses ways of minimis- 
ing disruption and avoiding individual staff problems-but he has nothing to say about the instinctive defence of their institutions by staff of all grades and disciplines. Professor Harold Ellis presents one view of the proposals as seen from the Westminster Hospital at p 691: clearly all closures will be fought to the last ditch.

Opposition will come, too, from medical teachers who dispute the virtues of size. A medical school with an intake of several hundred students each year will be very different in character from the London tradition. No one doubts that above a certain limit more means worse; the dispute is about the numbers at which that limit is reached. Furthermore, if economies in university spending are to result from the Flowers proposals the number of medical teachers will presumably be reduced. That means either that staff-student ratios will deteriorate or that more teaching will have to be done by NHS staff with honorary contracts-not necessarily a bad thing, but a policy on which those staff have not been consulted. The BMA's Medical Academic Staff Committee will be examining this part of the Flowers Report with special care.

These fundamental aspects of the solutions offered by the Flowers Committee may divert attention from criticisms of their details - and by tackling so much simultaneously London University may be hoping that the protests from each institution under threat will merge into an unintelligible babel. The crucial constraint will be the timing. By now everyone who has worked in the NHS or in British universities knows the debilitating effects of prolonged uncertainty on both buildings and people. The problems are real-a change in the population of central London, an excess of beds there, and too little money. These are serious proposals that warrant debate and will need modification, but some time limit will have to be imposed.

${ }^{1}$ University of London. London medical education-a new framework. Report of a Working Party on Medical and Dental Teaching Resources, chairman Lord Flowers. London: University of London, 1980.

2 London Health Planning Consortium. Towards a balance: a framework for acute hospital services in London reconciling service with teaching needsa discussion document. Lonçon: London Health Planning Consortium, 1980.

${ }^{3}$ DHSS. Sharing resources for health in England: report of the Resource Allocation Working Party. London: HMSO, 1976.

${ }^{4}$ Royal Commission on Medical Education 1965-8. Report. London: HMSO, 1968.

\section{Polypharmacy in rheumatic diseases}

Traditionally polypharmacy is condemned: in reality it is widely practised. Many patients with arthritis receive more than one non-steroidal anti-inflammatory agent simultaneously or else one of these drugs ("for stiffness") with an analgesic ("for pain"). Evidence of the synergistic value of these combinations is often lacking. Claims have been made $^{1}$ for synergism between naproxen and aspirin in rheumatoid arthritis, but others have failed to show this synergism. ${ }^{2}$ Furthermore, serum indomethacin concentrations may even be lowered by giving salicylates at the same time, ${ }^{34}$ though again there are conflicting reports. ${ }^{25}$ Nor are serum concentrations of a drug necessarily a guide to clinical improvement. A recent study from New Zealand ${ }^{6}$ of the possible clinical and pharmacokinetic interaction between aspirin and ibuprofen in rheumatoid arthritis showed a weak additive clinical effect between $1600 \mathrm{mg}$ ibuprofen and $3600 \mathrm{mg}$ aspirin daily, though not with lower doses of the drugs. Ibuprofen had no effect on serum concentrations of salicylate, but salicylate reduced the serum concentration of ibuprofen without affecting its elimination half life. Altered absorption of ibuprofen, protein binding, or possibly both may have been responsible for the fall. The patients did not appear to suffer clinically.

While a plethora of new non-steroidal anti-inflammatory agents has been introduced, little attention has been paid to the most efficient use of the old ones. Patients are seldom advised about the relation of dosage to meals, and doctors forget that half lives of drugs tend to be longer in older patients, so that less frequent administration may be reasonable in rheumatic diseases of the elderly. Monitoring of serum concentrations, useful as a test of compliance as well as drug availability, is becoming more easily available to rheumatologists. Few practitioners realise, for example, that phenylbutazone with a half life of around 70 hours needs to be tried for at least two weeks before being discarded as "ineffective." Equally, pharmacokinetics should not override the clinical picture as testified by the rapid response of patients with gout to phenylbutazone in spite of its long half life. In rheumatic diseases, drug concentrations in synovial fluid and the tissues are likely to be more relevant than those in the serum.

The non-steroidal anti-inflammatory agents may be grouped generically by their clinical structure into a few families, yet few studies have attempted to correlate clinical improvement with plasma profiles when drugs from two different families are given simultaneously-the rational approach to polypharmacy. The treatment of epilepsy has been revolutionised by neurologists learning to replace multiple drug treatment with closely monitored use of a single drug, and all rheumatologists would agree that the difficulty is matching the right drug to the right disease in the right patient.

While polypharmacy among non-steroidal anti-inflammatory agents might be usefully trimmed, the concept of polypharmacy in the specific long-term treatment of rheumatoid arthritis is more exciting. Traditionally, drugs such as Dpenicillamine and gold are given in isolation. Analogy with diseases such as ulcerative colitis and lymphoma, both chronic diseases with autoimmune aspects, suggests there may be a case for initiating a remission with one agent and subsequent maintenance treatment with a second. So far, however, the similarity of the side effects of gold and Dpenicillamine has prevented these drugs being used in rapid succession-and, indeed, studies in patients where the drugs have been given with a respectable interval between them suggest that there may still be some accumulation of adverse reactions. ${ }^{7}$

Some patients with rheumatoid arthritis are started on steroids pending subsequent transfer to a "steroid-sparing agent" such as D-penicillamine in order to gain rapid improvement when immediate relief of symptoms is required, though many rheumatologists believe the possible side effects of prednisolone do not justify its dramatic results. Prednisolone does, however, reduce the amount of cyclophosphamide required to produce remission and regression of radiological erosions in rheumatoid arthritis. ${ }^{8}$ Whether it "potentiates" other drugs in this manner is not yet known. Old drugs recently claimed to have specific antirheumatoid properties include sulphasalazine, ${ }^{9}$ oral gold, ${ }^{10}$ dapsone, ${ }^{11}$ 